\title{
Electrowetting diminishes contact line friction in molecular wetting
}

\author{
Petter Johansson $\odot$ and Berk Hess* \\ Science for Life Laboratory, Department of Applied Physics, and Swedish e-Science Research Center, KTH \\ Royal Institute of Technology, Stockholm SE-106 91, Sweden
}

(Received 20 December 2019; accepted 12 June 2020; published 29 June 2020)

\begin{abstract}
We use large-scale molecular dynamics to study the dynamics at the three-phase contact line in electrowetting of water and electrolytes on no-slip substrates. Under the applied electrostatic potential the line friction at the contact line is diminished. The effect is consistent for droplets of different sizes as well as for both pure water and electrolyte solution droplets. We analyze the electric field at the contact line to show how it assists ions and dipolar molecules to advance the contact line. Without an electric field, the interaction between a substrate and a liquid has a very short range, mostly affecting the bottom, immobilized layer of liquid molecules which leads to high friction since mobile molecules are not pulled towards the surface. In electrowetting, the electric field attracts charged and polar molecules over a longer range, which diminishes the friction.
\end{abstract}

DOI: 10.1103/PhysRevFluids.5.064203

\section{INTRODUCTION}

Recent developments in the study of liquid droplets spreading on surfaces have shown that the dynamics can be limited by a mixture of inertia and viscous and contact line energy dissipation. The term that dominates the process can be determined from the balance of nondimensional Ohnesorge numbers, which relate viscous friction to surface tension and inertial forces [1]. These are given by $\mathrm{Oh} \equiv \mu / \sqrt{\rho \gamma R}$ and $\mathrm{Oh}_{\mathrm{f}} \equiv \mu_{\mathrm{f}} / \sqrt{\rho \gamma R}$, where $\mu$ and $\rho$ are the liquid viscosity and density, respectively, $\gamma$ is the liquid-vapor surface tension, $R$ is the initial droplet radius, and $\mu_{\mathrm{f}}$ is a contact line friction parameter which has units of viscosity. As Do-Quang et al. [1] show for the initial rapid wetting phase, $\mathrm{Oh} \gg 1$ correlates with viscous forces dominating the wetting dynamics over surface tension. Similarly, when $\mathrm{Oh}_{\mathrm{f}} \gg 1$ contact line friction does. This is the case for certain hydrogen bonding [2,3] or microstructured substrates [4].

Surprisingly, applying an electric potential to a droplet, a phenomenon covered under the umbrella of electrowetting (see [5,6] for comprehensive reviews and [7-9] for some recent experiments), diminishes contact line dissipation [10]. Moreover, a recent study using lithographed substrates shows that under electrowetting the wetting can shift from a line friction dominated to a viscously dominated regime [11]. The authors refer to this as an electrostatic cloaking of the microscopic substrate features.

It is not yet known how the contact line advancement is affected to reduce the influence of line friction under these conditions. This is not helped by the fact that although models of contact line friction have been proposed for different length scales (including our previous work on molecular wetting [3] and of Perrin et al. on microscopic [12]), we lack a holistic understanding of the phenomena.

\footnotetext{
*hess@kth.se
}

Published by the American Physical Society under the terms of the Creative Commons Attribution 4.0 International license. Further distribution of this work must maintain attribution to the author(s) and the published article's title, journal citation, and DOI. Funded by Bibsam. 


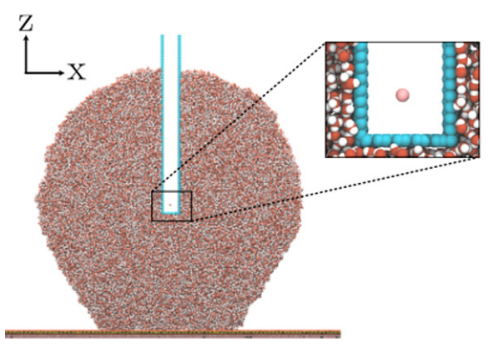

(a)

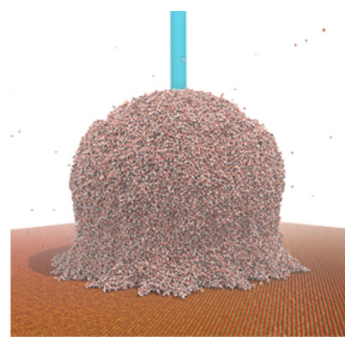

(b)

FIG. 1. Electrowetting system with a 15-nm-radius water droplet. (a) Two-dimensional slice of the system, highlighting the electrode charge. Below the substrate is a layer of countercharges. (b) System during an electrowetting experiment. (Figures were created using MATPLOTLIB [13].)

In this paper we investigate how electrowetting affects contact line friction on a molecular level in the rapid wetting regime, using computer molecular dynamics simulations of pure water and an electrolyte solution. We consider how the cloaking effect relates to our previously proposed model of molecular line friction.

\section{METHOD}

Electrowetting systems were constructed for molecular simulations with three base components: a planar substrate, a liquid droplet, and an electrode (Fig. 1). The atomic substrate used is silicalike $\mathrm{SiO}_{2}$ electrostatic quadrupoles set in a monolayer with fcc packing. The equilibrium contact angle $\theta_{0}$ is set by tuning the atomic charges while keeping the molecules neutral, with $q_{\mathrm{Si}}=-2 q_{\mathrm{O}}$. We use three sets of charges with equilibrium contact angles of $70^{\circ}, 90^{\circ}$, and $110^{\circ}$. For the liquid droplet we use both pure water (PW), which hydrogen bonds with the $\mathrm{SiO}_{2}$ quadrupoles, and a $\mathrm{KCl}$ electrolyte solution $[14,15]$ at a concentration of $3 \mathrm{M}$. Since water hydrogen bonds to the silica substrate it is effectively a no-slip substrate [2]. The water model is the extended simple point charge model [16], which has $\rho=990 \mathrm{~kg} \mathrm{~m}^{-3}, \gamma=5.8 \times 10^{-2} \mathrm{~Pa} \mathrm{~m}$, and $\mu=8.8 \times 10^{-4} \mathrm{Pas}$ at the simulated system temperature of $300 \mathrm{~K}$. The initial droplet radii $R_{0}$ are 7.5 and $15 \mathrm{~nm}$, with around 59000 and 470000 liquid molecules, respectively. As an electrode a neutral carbon nanotube with a radius of $1 \mathrm{~nm}$ was used, with its interaction parameters tuned to give a $90^{\circ}$ contact angle.

An electric potential difference $U$ is created by putting a single fixed charge $Q$ close to the bottom of the electrode and $n$ opposite charges $q_{n}$ below the planar substrate such that $n q_{n}=-Q$. These lower charges are free to move in the plane below the substrate. This creates a nonhomogeneous electric field directed towards (or away from) the lower end of the electrode. For the 7.5-nm droplets we used $n=1000$ and $Q=200 e$ and for the 15-nm droplets $n=4000$ and $Q= \pm 400 e$, where $e$ is the electron charge. The larger droplet was run for both a positive and negative potential by switching the charge signs.

The charge values were selected to produce a large change in contact angle $\theta_{0}^{*}$ from the static contact angle $\theta_{0}$. The electrostatic potential difference $U$ was then measured from the surface to the electrode using the PMEPOT plugin of VMD $[17,18]$. Contact angles $\theta_{0}$ and $\theta_{0}^{*}$ and potentials $U$ are reported in Table I.

Note that experiments of electrowetting display a saturation of the contact angle $\theta_{0}^{*}$ for increasing potentials $U$. We have not precisely characterized the saturation for our systems, but the experiments appear to be in the saturated regime. A comparison experiment of our $15-\mathrm{nm}$ pure water droplet with $1 / 2$ the applied potential $U$ does not result in $1 / 4$ of the force at the contact line as the YoungLippmann relation (1) predicts, but instead approximately $1 / 2$. As we will discuss later, the water dipole ordering is high at the contact line, which leads to a nonlinear dielectric response [19] that could explain a large part of the saturation. However, analyzing this is outside the scope of this paper. 
TABLE I. Initial and final droplet states of simulations. Here $R_{0}$ is the initial droplet radius, $\theta_{0}$ is the static contact angle for no electric potential, and $\theta_{0}^{*}$ is the static contact angle for the applied potential $U$.

\begin{tabular}{lcccc}
\hline \hline Droplet & $R_{0}(\mathrm{~nm})$ & $\theta_{0}(\mathrm{deg})$ & $\theta_{0}^{*}(\mathrm{deg})$ & $U(\mathrm{~V})$ \\
\hline pure water & 15 & 110 & 65 & \pm 110 \\
pure water & 7.5 & 70 & 56 & 55 \\
pure water & 7.5 & 90 & 57 & 55 \\
pure water & 7.5 & 110 & 59 & 55 \\
$\mathrm{KCl}$ & 7.5 & 90 & 55 & 11 \\
\hline \hline
\end{tabular}

Simulations were performed using GROMACS 2018 [20] in double precision with a leapfrog integrator and time step of 2 fs. Short-range interactions were treated fully up to a cutoff of $0.9 \mathrm{~nm}$. Long-range electrostatic interactions were treated using the particle-mesh Ewald method, which has an infinite, periodic interaction range. Periodic boundary conditions were applied along the $x$ and $y$ axes and repulsive walls were placed at the simulation box edges along $z$ to contain particles in the system. We verified that the periodic boundary treatment does not significantly affect the results by increasing the periodic distances.

Contact angles $\theta(t)$ were measured for each output simulation frame at time $t$ using the approach introduced by Khalkhali et al. [21]. The wetting radius $r(t)$ was calculated using a radial density distribution of the bottom layer of water molecules, from its center.

\section{RESULTS AND DISCUSSION}

Wetting simulations were performed in two stages. First the droplets were allowed to relax to their equilibrium states on the substrates while the electrode and below-substrate atoms where uncharged. After equilibration, the charges were increased to their final values over $50 \mathrm{ps}$ using a sigmoid activation function. As the electric field is created, the droplet spreads out to a smaller equilibrium contact angle $\theta_{0}^{*}$ modeled by the Young-Lippmann relation

$$
\cos \theta_{0}^{*}=\cos \theta_{0}-\frac{c U^{2}}{2 \gamma}
$$

for the substrate capacitance per unit area $c$. Unlike a prior computational study of electrowetting on gold [22], no precursor film is present.

We record the base radius $r(t)$ and contact angle $\theta(t)$ starting from the fully applied field. These are presented in Fig. 2 with the final states given in Table I. The final state is reached quickly for all systems, although the $15-\mathrm{nm}$ and electrolyte droplets overshoot and retract to their final $\theta_{0}^{*}$ (not shown in the figure). This is particularly noticeable for the $\mathrm{KCl}$ system, which has extremely rapid dynamics. Contact line friction is measured by how much the contact line speed $v$ is damped compared to what we would expect from the Young driving force [10,23,24]. With $\mu_{\mathrm{f}}$ being the friction parameter, the velocity is given by $v=\gamma\left(\cos \theta_{0}^{*}-\cos \theta\right) / \mu_{\mathrm{f}}$. Since we can calculate $v$ from the spreading radius $r(t)$, we estimate the friction parameter for our data sets using this relation. These results are presented in Fig. 3, using only data from the rapid wetting phase. Due to the influence of thermal fluctuations, the $7.5-\mathrm{nm} 110^{\circ}$ droplet gives a relatively high error, but its value is still within the error of the other systems including, notably, both $15-\mathrm{nm} 110^{\circ}$ droplets. The dynamic evolutions of $\mu_{\mathrm{f}}(t)$ and $v(\cos \theta)$ are both available in the Supplemental Material [25].

Consistent with previous studies, we see that the line friction is very low for our electrowetting systems and that all systems have very similar amounts of friction, $0.2 \mu-0.8 \mu$. We have previously shown [3] that spontaneous wetting of pure water on the $70^{\circ}$ substrate without an electric field gives a line friction that increases from $2 \mu$ to $8 \mu$ as the equilibrium is approached, while its average is 


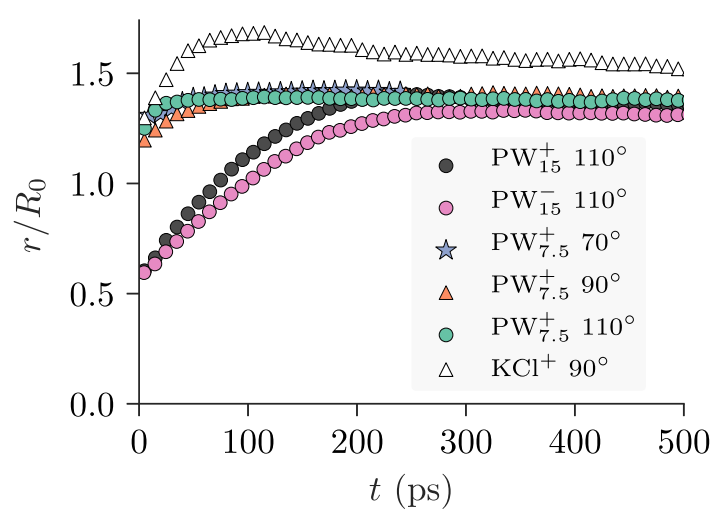

(a)

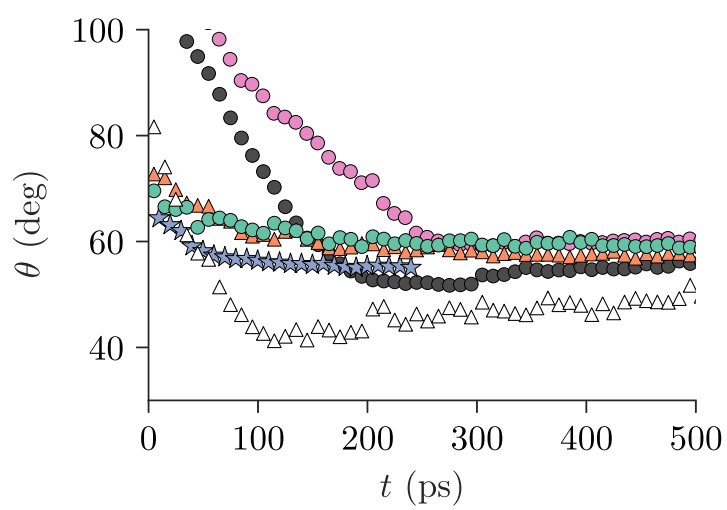

(b)

FIG. 2. (a) Spreading base radius and (b) contact angle for all systems. The droplet radius is noted for the pure water systems. The applied potential sign is shown for all systems.

below $0.5 \mu$ with the electric field. Electrowetting thus decreases the line friction on a molecular level by an order of magnitude, for molecularly flat substrates.

It is interesting to relate our results to the regime map of the Ohnesorge numbers $\mathrm{Oh}$ and $\mathrm{Oh}_{\mathrm{f}}[1]$, as discussed earlier. For the $15-\mathrm{nm}$ droplet we have $\mathrm{Oh}=0.94$. With a friction factor of $\mu_{\mathrm{f}}=5 \mu$,



FIG. 3. Measured mean contact line friction during the rapid spreading phase for all systems. Vertical bars mark \pm 1 standard error. 
(a)

(b)

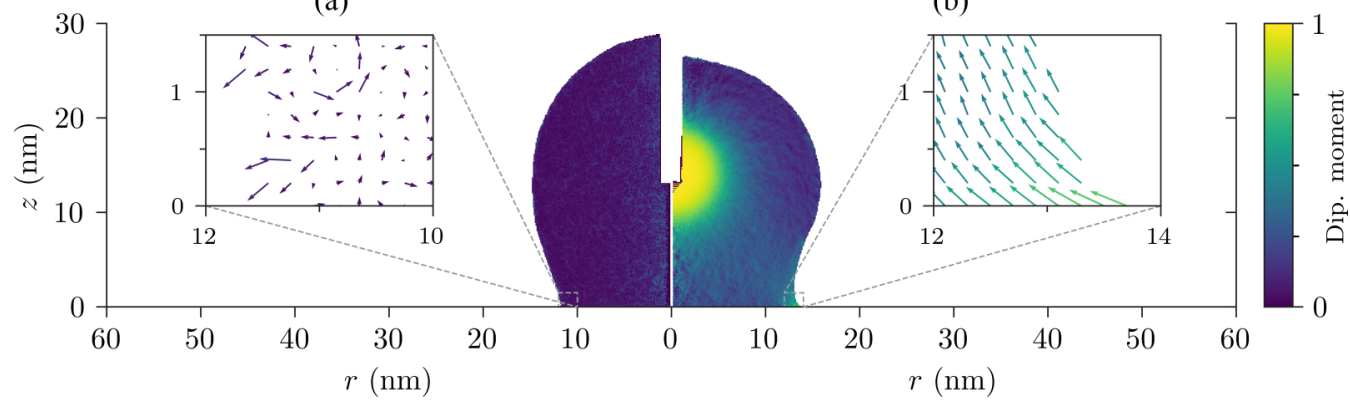

FIG. 4. Radial distribution of measured dipole orientation inside droplets during (a) spontaneous wetting and (b) electrowetting of pure water. Insets show the averaged dipole direction and magnitude at contact lines where the arrows are scaled by a factor of 5 for the spontaneous view. The moment is normalized by the dipole moment of the water molecule.

as previously measured for spontaneous wetting [3], the same system gives $\mathrm{Oh}_{\mathrm{f}}=4.7$. This places the system in the regime where line friction dominates the contact line advancement in spontaneous wetting. With $\mu_{\mathrm{f}}<0.5$, measured for electrowetting in Fig. 3, we shift into the regime where inertial or viscous forces dominate $\left(\mathrm{Oh}_{\mathrm{f}}<1\right)$.

We finally consider where this decrease in line friction originates. Our previous model [3] of contact line friction on no-slip substrates proposes that it is an effect of a molecule having to cross an energy barrier $\Delta E$ of order $k_{\mathrm{B}} T$ in a thermal fluctuation to reach the substrate and advance the contact line, which causes significant friction. This energy barrier stems from the internal hydrogen bonding network between water molecules at the contact line and is given by

$$
\Delta E=a(\cos \theta+0.5 \sin \theta)^{2},
$$

transformed from the reported equation by introducing the Boltzmann factor $k_{\mathrm{B}} T$ into the exponential (due to it being a thermally activated process) and using some trigonometric identities. At $T=300 \mathrm{~K}$ the value $a=1.1 k_{\mathrm{B}} T$ matched the same silica substrate used here.

That this barrier creates friction comes from another observation: The effective force between the substrate and water has an extremely short range, barely affecting more than the bottommost layer of water molecules [2]. A water molecule cannot experience the attraction from the surface until it gets very close, which means that there is little to no assist in crossing the barrier. In electrowetting this situation changes greatly at the contact line. The potential difference creates an electric field $\mathbf{E}=-\nabla U$. Ions, as in our $\mathrm{KCl}$ electrolyte, are directly attracted along this field, leading to a direct assist in crossing the energy barrier.

For neutral molecules, like water, the situation is more complicated. There is no net attraction in a homogeneous electric field but polar molecules with moment $\mathbf{p}$ experience a force $\mathbf{F}_{p}=(\mathbf{p} \cdot \nabla) \mathbf{E}$ if the field is nonuniform. As water is a dipole, this force will be present at the contact line, where the electric field changes abruptly.

Our molecular data allow us to measure both $\mathbf{E}$ and $\mathbf{p}$ throughout our system. Figure 4 shows an example radial distribution of the polarization inside a $15-\mathrm{nm}$ pure water droplet during the spreading phase, where the data are averaged over $10 \mathrm{ps}$. To estimate the influence of $\mathbf{F}_{p}$ we calculate it for the same system but after it has reached an equilibrium state with fixed contact angle $\theta_{0}=65^{\circ}$. We calculate it along the droplet interface, averaging over the range inside the radial distribution where the mass is $40-60 \%$ of the bulk value.

Figure 5 shows the radial components $F_{r}$ and $F_{z}$ of $\mathbf{F}_{p}$ at different heights $z$ from the top oxygen atom in the $\mathrm{SiO}_{2}$ substrate. We also show a term $F_{45^{\circ}}$, which is the force projected along a unit vector pointing towards the surface at an angle $45^{\circ}$. This is to (roughly) represent the force pointing along a path towards the substrate. The $z$ component of the force is very high close to the surface, owing 


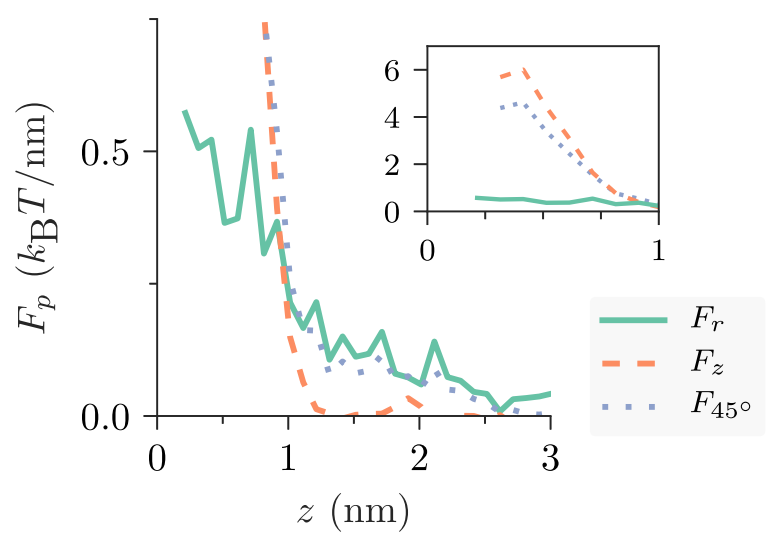

FIG. 5. Radial components of the dipole force $\mathbf{F}_{p}$ for water molecules at height $z$ above the top substrate atoms. Here $F_{z}$ is positive for a force directed towards the substrate.

to the large gradient of the $z$ component of the electric field in that range. The radial component has a longer tail.

How does this compare to the energy barrier $\Delta E$ ? With $\theta=65^{\circ}$ and $a=1.1 k_{\mathrm{B}} T$, Eq. (2) gives $\Delta E=0.84 k_{\mathrm{B}} T$ and with $\theta=90^{\circ}$ it is $\Delta E=0.28 k_{\mathrm{B}} T$. Since it is unclear which path a water molecule will move when advancing the contact line, we cannot directly calculate its energy gain in the electric field, but even movements of a single water molecule diameter $(\sim 0.25 \mathrm{~nm})$ with the $F_{r}$ component yield an estimate of $0.17 k_{\mathrm{B}} T$, a significant part of the energy barrier at $90^{\circ}$. Integrating $F_{45}$ o up to $1 \mathrm{~nm}$ gives $1.75 k_{\mathrm{B}} T$. The dipole force term is thus significant over a range of at least 1 $\mathrm{nm}$, which will assist molecules in crossing the energy barrier, if not remove it. We highlight the contrast to the Young force in Fig. 6.

Some additional effects related to the polarization at the contact line may contribute to the line friction decrease. As seen in Fig. 4, the water dipoles are highly ordered at the contact line due to the electric field. This ordering may by itself affect the ease of contact line advancement. We analyze two properties which are affected by the ordering: the hydrogen bonding network of molecules which have to pass the barrier and how bulk viscosity is changed by the high shear stress and ordering.

For the hydrogen bond network we identify the water molecules which are about to advance the contact line by rolling down from the second water layer. The number of hydrogen bonds

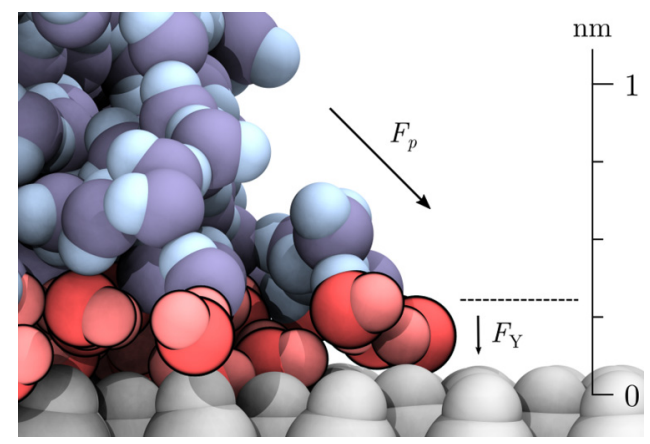

FIG. 6. The Young force $F_{\mathrm{Y}}$ between the substrate and liquid mostly affects the bottom water layer (shaded red with thick outlines). The dipole force $F_{p}$ is shown as a longer range, reaching upper molecule layers (shaded blue). 
between these and the surrounding molecules are then counted for the states shown in Fig. 4 by using the HBOND tool provided by GROMACS with default settings. For the spontaneous wetting case an average of 2.6 hydrogen bonds were identified per molecule. For the electrowetting case with high dipole ordering we counted 2.0 hydrogen bonds per molecule.

Thus the energy barrier for a molecule rolling to the contact line is lower by up to 0.6 times the free energy of a hydrogen bond $\Delta G$. For liquid water at room temperature $\Delta G=5.7 \mathrm{~kJ} / \mathrm{mol}=$ $2.3 k_{\mathrm{B}} T$ [26], which gives $0.6 \Delta G=1.4 k_{\mathrm{B}} T$. Note that this number may be in part due to the high ordering and in part due to the transition state having been modified by the electric field and the dipole force $\mathbf{F}_{p}$. We can measure the effect but not the cause.

Finally, we consider shear thinning, which may occur for the high shear rates at the contact line during electrowetting. To quantify this effect, we used a simple Couette flow shear setup. We do not observe a significant change in viscosity due to the shear. However, if we additionally apply an electric field which creates $75 \%$ dipole ordering, the viscosity becomes anisotropic. The viscosity with shearing in the direction of the electric field increases by $30 \%$. This means that local viscous dissipation will decrease due to the high ordering, but the change is quantitatively much smaller than the weakened hydrogen bonding network described above.

The droplets used here are several order of magnitude smaller than typical droplet sizes. When scaling the droplet size, the voltage can be kept constant to maintain the same contact angle. The electric field drops sharply at the liquid interface, at a molecular length scale [27]. As the reduction in contact line friction is due to the strong electric field and its gradient at the contact line, it will be present for larger droplets, consistent with experiments.

We want to note that this purely molecular effect is not the first seen in electrowetting using MD simulations. Daub et al. have reported on the asymmetry of water molecules yielding dynamics, which depends on the sign of the applied potential [28], and Yuan and Zhao on how the precursor film (not present here due to the quadrupole substrate) forms a molecular network with unique transport properties [22]. In addition, Liu et al. observed that contact angle saturation occurs as individual molecules are pulled out of the contact line to shield the rest of the interface from the applied potential [29], an effect that is clearly visible for macroscopic systems. Along with these, our work again highlights how the molecular nature of liquids can influence dynamic and macroscopic behavior.

\section{CONCLUSION}

Through molecular simulation we have verified that application of an electric field decreases contact line friction of smooth molecular systems, consistent with experiments on Teflon-coated and microstructured substrates [10,11]. It is consistent for systems of pure water with different initial radii and an electrolyte. The decrease in line friction is of one order of magnitude, which may shift systems from being dominated by line friction to another dynamic regime.

We have identified two sources at the molecular level for the decrease in friction. The first and main source is that the interaction range between the surface and liquid increases, which largely avoids the high-energy barrier that a single layer of water molecules has to cross to advance the contact line. For electrowetting this comes from the electric field and its gradient, which is strong in a region of a few nanometers around the contact line. The other effect is the high ordering of dipoles at the contact line, also due to the strong electric field. This changes the local hydrogen bond network, decreasing the overall number of bonds that water molecules have to break in order to advance the contact line.

Several open questions remain. Most of the above discussion relates to single water molecules independently advancing the contact line; however, wetting is a more collective phenomena, where a single advancing molecule pulls along one or more other molecules. Such correlated movements could further influence how we view line friction, but are difficult to study due to the thermal velocity being much higher than the contact line velocity. 


\section{ACKNOWLEDGMENTS}

We would like to thank J. Shiomi for discussions leading to this work. The research was made possible by funding from the Swedish Research Council (Grant No. 2014-4505). Simulations were performed on resources provided by the Swedish National Infrastructure for Computing (Grants No. 2018/1-22 and No. 2019/1-22) at the PDC Center for High Performance Computing.

[1] M. Do-Quang, J. Shiomi, and G. Amberg, When and how surface structure determines the dynamics of partial wetting, Europhys. Lett. 110, 46002 (2015).

[2] P. Johansson, A. Carlson, and B. Hess, Water-substrate physicochemistry in dynamic wetting, J. Fluid Mech. 781, 695 (2015).

[3] P. Johansson and B. Hess, Molecular origin of contact line friction in dynamic wetting, Phys. Rev. Fluids 3, 074201 (2018).

[4] J. Wang, M. Do-Quang, J. J. Cannon, F. Yue, Y. Suzuki, G. Amberg, and J. Shiomi, Surface structure determines dynamic wetting, Sci. Rep. 5, 8474 (2015).

[5] F. Mugele and C.-J. Baret, Electrowetting: From basics to applications, J. Phys.: Condens. Matter 17, R705 (2005).

[6] Y.-P. Zhao and Y. Wang, Fundamentals and applications of electrowetting, Rev. Adhesion Adhesives 1, 114 (2013).

[7] C. V. Brown, G. G. Wells, M. I. Newton, and G. McHale, Voltage-programmable liquid optical interface, Nat. Photon. 3, 403 (2009).

[8] G. McHale, C. V. Brown, and N. Sampara, Voltage-induced spreading and superspreading of liquids, Nat. Commun. 4, 1605 (2013).

[9] D. J. Lomax, P. Kant, A. T. Williams, H. V. Patten, Y. Zou, A. Juel, and R. A. W. Dryfe, Ultra-low voltage electrowetting using graphite surfaces, Soft Matter 12, 8798 (2016).

[10] C. Decamps and J. De Coninck, Dynamics of spontaneous spreading under electrowetting conditions, Langmuir 16, 10150 (2000).

[11] S. Nita, M. Do-Quang, J. Wang, Y.-C. Chen, Y. Suzuki, G. Amberg, and J. Shiomi, Electrostatic cloaking of surface structure for dynamic wetting, Sci. Adv. 3, e1602202 (2018).

[12] H. Perrin, R. Lhermerout, K. Davitt, E. Rolley, and B. Andreotti, Defects at the Nanoscale Impact Contact Line Motion at All Scales, Phys. Rev. Lett. 116, 184502 (2016).

[13] J. D. Hunter, Matplotlib: A 2D graphics environment, Comput. Sci. Eng. 9, 90 (2007).

[14] S. Weerasinghe and P. E. Smith, A Kirkwood-Buff derived force field for sodium chloride in water, J. Chem. Phys. 119, 11342 (2003).

[15] B. Hess and N. F. A. van der Vegt, Cation specific binding with protein surface charges, Proc. Natl. Acad. Sci. USA 106, 13296 (2009).

[16] H. J. C. Berendsen, J. R. Grigera, and T. P. Straatsma, The missing term in effective pair potentials, J. Phys. Chem. 91, 6269 (1987).

[17] A. Aksimentiev and K. Schulten, Imaging $\alpha$-hemolysin with molecular dynamics: Ionic conductance, osmotic permeability, and the electrostatic potential map, Biophys. J. 88, 3745 (2005).

[18] W. Humphrey, A. Dalke, and K. Schulten, VMD: Visual molecular dynamics, J. Mol. Graph. 14, 33 (1996).

[19] H. E. Alper and R. M. Levy, Field strength dependence of dielectric saturation in liquid water, J. Phys. Chem. 94, 8401 (1990).

[20] M. J. Abraham, T. Murtola, R. Schulz, S. Páll, J. C. Smith, B. Hess, and E. Lindahl, Gromacs: High performance molecular simulations through multi-level parallelism from laptops to supercomputers, SoftwareX 1-2, 19 (2015).

[21] M. Khalkhali, N. Kazemi, H. Zhang, and Q. Liu, Wetting at the nanoscale: A molecular dynamics study, J. Chem. Phys. 146, 114704 (2017). 
[22] Q. Yuan and Y.-P. Zhao, Precursor Film in Dynamic Wetting, Electrowetting, and Electro-ElastoCapillarity, Phys. Rev. Lett. 104, 246101 (2010).

[23] T. D. Blake and J. M. Haynes, Kinetics of liquid/liquid displacement, J. Colloid Interface Sci. 30, 421 (1969).

[24] P. Yue and J. J. Feng, Wall energy relaxation in the Cahn-Hilliard model for moving contact lines, Phys. Fluids 23, 012106 (2011).

[25] See Supplemental Material at http://link.aps.org/supplemental/10.1103/PhysRevFluids.5.064203 for a plot of $\mu_{f}(t)$ and a plot of $v(\cos \theta)$.

[26] D. van der Spoel, P. J. van Maaren, P. Larsson, and N. Tîmneanu, Thermodynamics of hydrogen bonding in hydrophilic and hydrophobic media, J. Phys. Chem. B 110, 4393 (2006).

[27] V. Ballenegger and J.-P. Hansen, Dielectric permittivity profiles of confined polar fluids, J. Chem. Phys. 122, 114711 (2005).

[28] C. D. Daub, D. Bratko, K. Leung, and A. Luzar, Electrowetting at the nanoscale, J. Phys. Chem. C 111, 505 (2007).

[29] J. Liu, M. Wang, S. Chen, and M. O. Robbins, Uncovering Molecular Mechanisms of Electrowetting and Saturation with Simulations, Phys. Rev. Lett. 108, 216101 (2012). 